Cinémas

Revue d'études cinématographiques

Journal of Film Studies

\title{
La cinématographie comme dispositif (du) spectaculaire
}

\section{Frank Kessler}

Volume 14, numéro 1, automne 2003

Dispositif(s) du cinéma (des premiers temps)

URI : https://id.erudit.org/iderudit/008956ar

DOI : https://doi.org/10.7202/008956ar

Aller au sommaire du numéro

\section{Éditeur(s)}

Cinémas

ISSN

1181-6945 (imprimé)

1705-6500 (numérique)

Découvrir la revue

Citer cet article

Kessler, F. (2003). La cinématographie comme dispositif (du) spectaculaire. Cinémas, 14(1), 21-34. https://doi.org/10.7202/008956ar

\section{Résumé de l'article}

En partant du concept du dispositif tel qu'il a été défini dans les années 1970 par Jean-Louis Baudry notamment, cet article cherche à le redéfinir de deux façons : d'abord en tenant compte de l'évolution diachronique du dispositif remettant ainsi en question le mythe de son unicité - , puis en privilégiant une approche pragmatique historique - abandonnant ainsi la perspective métapsychologique traditionnelle. Les débuts de la cinématographie sont mis à contribution en vue de mettre en évidence deux formes de dispositif ayant précédé celui du cinéma institutionnel : la cinématographie comme dispositif spectaculaire, dans le cadre duquel c'est la capacité de la machine de prendre et de reproduire du mouvement qui prédomine ; et la cinématographie comme dispositif du spectaculaire, où c'est le spectacle filmé lui-même qui constitue l'attraction principale. 


\section{La cinématographie comme

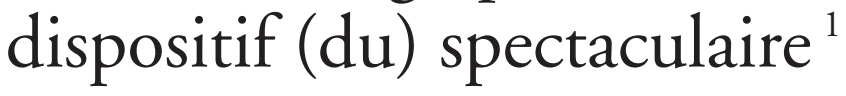

\section{Frank Kessler}

\section{RÉSUMÉ}

En partant du concept du dispositif tel quil a été défini dans les années 1970, par Jean-Louis Baudry notamment, cet article cherche à le redéfinir de deux façons: d'abord en tenant compte de l'évolution diachronique du dispositif — remettant ainsi en question le mythe de son unicité - , puis en privilégiant une approche pragmatique historique - abandonnant ainsi la perspective métapsychologique traditionnelle. Les débuts de la cinématographie sont mis à contribution en vue de mettre en évidence deux formes de dispositif ayant précédé celui du cinéma institutionnel: la cinématographie comme dispositif spectaculaire, dans le cadre duquel c'est la capacité de la machine de prendre et de reproduire du mouvement qui prédomine; et la cinématographie comme dispositif du spectaculaire, où c'est le spectacle filmé lui-même qui constitue l'attraction principale.

\section{ABSTRACT}

Taking as a point of departure the concept of the apparatus, particularly as defined in the 1970's by Jean-Louis Baudry, this article seeks to redefine it in the following ways: first, while accounting for the diachronic evolution of the apparatus, it calls into question the myth of its unicity; next, while privileging a pragmatic historical approach, it abandons the traditional metapsychological perspective. The beginnings of cinematography are reviewed in order to highlight two forms of the apparatus which preceded that of institutionalized film: cinematography as spectacular apparatus, in which the dominant element is the capacity of the machine to take and to 
reproduce movement; and cinematography as apparatus of the spectacular, in which it is the filmed spectacle itself that constitutes the principal attraction.

"Un média naît toujours deux fois..." - telle est la thèse élaborée par André Gaudreault et Philippe Marion, thèse annoncée dans le titre même de leur article paru en 2000. Dans une publication plus récente, les deux auteurs explicitent les principes de cette théorie de la "double naissance" des médias de la manière suivante:

$[\mathrm{N}]$ otre modèle repose sur une gradation à trois temps que nous avons identifiés par trois termes situés dans un même champ sémantique, mais auxquels nous avons donné un coefficient connotatif spécifique. Ces trois termes sont: apparition, émergence et avènement. L'histoire du cinéma des premiers temps nous ferait donc ainsi passer, successivement, de l'apparition d'un dispositif technique (une technologie), les machines à vues, à l'émergence d'un dispositif socioculturel, celui des vues animées, puis à l'avènement d'une institution, celle du cinéma (Gaudreault et Marion 2001, p. 34 ce sont les auteurs qui soulignent).

La notion de dispositif apparaît à deux reprises dans le passage que l'on vient de citer, prenant alors deux sens légèrement différents: d'un côté elle renvoie à la technologie cinématographique, c'est-à-dire à la capacité de la machine de capter et de restituer le mouvement, et de l'autre côté elle désigne la dimension socioculturelle du média. Et l'on pourrait ajouter que, en effet, ces deux acceptions de la notion apparaissent aujourd'hui assez couramment dans de nombreux écrits ayant trait au cinéma des premiers temps.

Or, en tenant compte des discussions métathéoriques en études cinématographiques des vingt dernières années, cela semble quelque peu étonnant: la théorie d'inspiration psychanalytique dite, justement, du dispositif ${ }^{2}$ (connue dans les pays anglophones sous le nom d'apparatus theory) n'a-t-elle pas été considérée jadis comme l'apogée d'un développement en matière d'études cinématographiques, théorie caractérisée par un manque absolu d'égard pour l'historicité de son objet — le 
cinéma en tant qu'institution et en tant que forme de spectacle? Ainsi, Thomas Elsaesser et Adam Barker (1990, p. 303) notent que "the enquiry [...] focusing on the fundamental nature of the cinematic apparatus [...] has tended to subsume all modes of cinematic spectatorship under Metz and Baudry's formulation of the Imaginary Signifier ${ }^{3}$ ".

La pertinence théorique de la notion de dispositif au sein d'une interrogation sur l'histoire du cinéma est donc loin d'être évidente. Il importe alors de l'explorer dans ses diverses acceptions afin que ressorte clairement son éventuelle utilité, voire sa productivité théorique pour la recherche historique.

Voici un rappel des grandes lignes de ladite théorie du dispositif. Dans leur Dictionnaire théorique et critique du cinéma, Jacques Aumont et Michel Marie expliquent tout d'abord que "le terme de dispositif désigne en mécanique la manière dont sont disposées les pièces et les organes d'un appareil, et par là, le mécanisme lui-même», et que la théorie freudienne reprend le terme pour rendre compte de l'organisation mentale de la subjectivité. C'est le sens qu'a retenu la théorie du cinéma pour décrire l'état psychique caractérisant le spectateur au cours de la projection d'un film. Aumont et Marie poursuivent:

Le dispositif est d'abord une organisation matérielle: les spectateurs perçoivent dans une salle obscure des ombres projetées sur un écran, produites par un appareil placé le plus souvent derrière leur tête. C'est l'«appareil de base» (Baudry), métonymie de l'ensemble de l'appareillage et des opérations nécessaires à la production d'un film et à sa projection, et donc pas seulement de la caméra et du projecteur proprement dits (Aumont et Marie 2001, p. 54).

Ce dispositif (que Baudry, on le sait, discute en évoquant le mythe de la caverne chez Platon ${ }^{4}$ ) provoque alors une régression du sujet, proche de celle du dormeur, répétant l'état postnatal, voire la vie intra-utérine, qui produit ce que Baudry appelle "l'effet cinéma».

Le cinéma provoque donc un retour vers un narcissisme relatif, vers une forme de réalité enveloppante dans laquelle les limites du corps propre du spectateur 
et sa relation à l'extérieur ne sont plus strictement précisées. Ainsi s'explique l'attachement du sujet psychique à l'image en général et plus particulièrement la forte identification exercée par le cinéma (Aumont et Marie 2001, p. 54-55).

En tenant compte d'autres travaux poursuivant ce type de réflexion, on doit remarquer que le régime discursif où cet effet cinéma se manifeste le plus efficacement, régime qui a dominé l'institution cinématographique jusqu'à aujourd'hui, c'est le film de fiction narratif-représentatif classique. Effectivement, les stratégies formelles déployées par celui-ci visent à maximaliser l'investissement psychique du spectateur dans la fiction.

En simplifiant les choses quelque peu, on pourrait dire que la théorie du dispositif propose en fin du compte une description $\mathrm{du}$ fonctionnement de l'institution cinématographique — avec tous les effets idéologiques que ceci implique par ailleurs - en termes d'une relation entre plusieurs facteurs:

- premièrement, une technologie matérielle produisant des conditions favorables à :

- deuxièmement, un certain positionnement du spectateur fondé sur des désirs inconscients auxquels correspond:

- troisièmement, une forme filmique institutionnalisée (le cinéma classique, pour aller vite) dont le mode d'adresse vise à garantir le maintien optimal de cette position spectatorielle, qualifiée le plus souvent de "voyeuriste ${ }^{5}$ ».

Même en laissant de côté la question de savoir dans quelle mesure on rencontre d'autres postures spectatorielles au sein de l'institution cinématographique, force est de constater que malgré toutes nos résistances envers les fondements psychanalytiques de cette théorie, et malgré le fait qu'elle ne tient pas compte explicitement de l'indéniable activité cognitive des spectateurs, elle a pu servir à de nombreuses études, en proposant une sorte de modèle général du fonctionnement du film de fiction narratif-représentatif dominant. À tel point que lorsque Tom Gunning cherche à donner une première définition de ce qu'il appelle le cinéma des attractions, il l'oppose explicitement, et presque terme à terme, à «l'effet cinéma» produit par le film narratif classique: 
What precisely is the cinema of attractions? [...] Contrasted to the voyeuristic aspect of narrative cinema analysed by Christian Metz, this is an exhibitionist cinema. An aspect of early cinema [...] is emblematic of this different relationship the cinema of attractions constructs with its spectator: the recurring look at the camera by actors. This action, which is later perceived as spoiling the realistic illusion of the cinema, is here undertaken with brio, establishing contact with the audience. From comedians smirking at the camera, to the constant bowing and gesturing of the conjurors in magic films, this is a cinema that displays its visibility, willing to rupture a self-enclosed fictional world for a chance to solicit the attention of the spectator (Gunning 1990, p. 57).

Autrement dit, le cinéma des attractions est un dispositif radicalement différent de celui du cinéma narratif classique, du moins en ce qui a trait au positionnement du spectateur et au mode d'adresse inhérent à la forme filmique. Et même si le dispositif matériel peut paraitre relativement proche de celui qui est discuté par Baudry, on ne saurait négliger les différences sur le plan des pratiques de présentation qui, en effet, sont moins aptes à produire cette absorption spectatorielle inhérente aux fictions cinématographiques classiques.

On voit dès lors que la notion de dispositif, conçue comme l'interrelation entre une technologie, un mode de présentation, un mode d'adresse, une forme filmique et un positionnement de l'instance spectateur, demande finalement à être "historicisée ${ }^{6}$ ". En abandonnant l'idée d'une prédisposition psychique fondamentale, inscrite pour ainsi dire ontologiquement dans le média en tant que tel et régissant la cinématographie dans son ensemble, on pourrait par ailleurs distinguer au cours de l'histoire du cinéma plusieurs dispositifs, chacun étant rendu spécifique par son historicité.

Ainsi, revenant à la notion de dispositif d'André Gaudreault et de Philippe Marion présentée plus haut, on peut formuler les choses un peu différemment: du point de vue proposé ici, chacun des paradigmes que les auteurs distinguent donne lieu à un dispositif différent, chacun de ces dispositifs ayant alors 
— forcément — à la fois une dimension technologique et une dimension socioculturelle, sans oublier les autres dimensions qu'on vient d'évoquer, à savoir la forme filmique et le mode d'adresse. Cela implique cependant une modification assez radicale de la visée théorique du concept de dispositif: on quitte le terrain de la métapsychologie du spectateur (ainsi que, fût-ce provisoirement, celui de la critique idéologique) pour se situer plutôt dans le domaine d'une pragmatique historique ${ }^{7}$.

\section{La cinématographie comme dispositif spectaculaire}

Quand Gaudreault et Marion parlent de l'«apparition d'un dispositif technique [...], les machines à vues", ils ne s'opposeraient sans doute pas à ce que l'on ajoute qu'il s'agit là d'une apparition qui, d'emblée, doit être située dans un environnement d'appareils et de spectacles optiques et visuels. Ou, pour utiliser une formule à la mode aujourd'hui, que les images en mouvement participent toujours déjà à une culture visuelle historiquement donnée. D'où cet autre phénomène constaté ailleurs par André Gaudreault (1997), selon lequel les diverses pratiques cinématographiques s'inscrivent toujours aussi dans d'autres séries culturelles. (Cela vaut aussi, bien évidemment, pour l'émergence des vues animées — on y reviendra.)

C'est le cas, par exemple, des différents types de Schnellseher développés par le photographe allemand Ottomar Anschütz ${ }^{8}$. Travaillant dans le contexte historique de la chronophotographie, mais sans avoir d'ambitions scientifiques en ce qui concerne l'étude du mouvement (à la différence d'un Muybridge ou d'un Marey), Anschütz produit, en effet, des photographies animées. Pour la commercialisation de ses travaux, il se sert de machines à vues basées sur les principes stroboscopiques du phénakistiscope ou du zootrope, et même, fût-ce brièvement, de la projection. Son Elektrischer Schnellseher est une machine à sous grâce à laquelle les spectateurs peuvent regarder une série d'images photographiques transparentes (vingt-quatre ou parfois moins), montées sur un disque. Anschütz reprend alors des technologies antérieures pour les adapter, modifier et perfectionner selon ses propres besoins. Le spectacle qu'il offre consiste principalement en la restitution 
photographique du mouvement, visant à l'émerveillement du spectateur face aux capacités de la machine. Dans ce contexte, il est hautement significatif qu'un des journalistes assistant à une séance de projection du Schnellseher appelle les photographies animées "Bewegungsbilder", c'est-à-dire "images de mouvement ${ }^{9}$ ".

On est alors au centre même de ce que Gaudreault et Marion (2001, p. 35-37) appellent le paradigme de la captation/ restitution. Les puissances de la technologie à capter le mouvement, la fidélité de sa restitution, l'ébahissement des spectateurs face à l'intensité bouleversante de ce qu'on appellera plus tard l'«impression de réalité», forment ainsi la dominante (pour emprunter ce terme au formalisme russe) du dispositif dans lequel s'inscrivent le Schnellseher ainsi que la plupart des autres machines, sinon toutes, avec lesquelles il entre en compétition. Ainsi, l'American Photographer du 16 mars 1894 précise dans un article consacré au kinétoscope d'Edison:

It will be remembered that the Electrical Wonder Company showed a large zoetrope with Anschütz pictures some little time back in the Strand, and these were a constant source of wonder and amazement to the uninitiated. Edison [...] will have some trouble to improve on these results (cité dans Rossell 1997, p. 17).

La presse de l'époque se fait d'ailleurs, avec insistance, l'écho d'une certaine fascination pour la force de l'illusion que produisent les diverses machines à vues, en raison notamment de leur précision dans la représentation de détails référentiels qui renforcent l'impression de la réalité: la fumée, les feuilles, le jeu des muscles, etc. La forme filmique (si l'on peut dire: il faudrait plutôt parler de "forme de photographie animée») correspondant à ce dispositif doit, justement, garantir avant tout la visibilité maximale de ces effets ${ }^{10}$.

Cela vaut aussi pour un cas qui, tout en appartenant à la même phase historique - celle de la novelty comme on l'appelle souvent - , pourrait être vu comme fonctionnant de manière différente, à savoir le cas des films des frères Skladanowsky. Présenté publiquement le $1^{\text {er }}$ novembre 1895 au Wintergarten de Berlin, le Bioskop des Skladanowsky s'inscrit d'emblée dans 
plusieurs séries culturelles. Du point de vue technologique, les Skladanowsky ont construit leur machine en partant de leurs expériences professionnelles et de leur double compétence dans les arts de la projection et de la photographie ${ }^{11}$. Du point de vue du contexte de présentation, par contre, c'est la série culturelle des arts du spectacle et, plus précisément, du théâtre des variétés qui intervient ici en tant que "dominante». Ainsi, une annonce du Wintergarten dans un journal berlinois précise que le Bioskop offre au public "un programme de théâtre de variétés complet en 15 minutes ${ }^{12}$ ".

Or, de ce point de vue, les films tournés par les Skladanowsky semblent clairement appartenir au paradigme de la monstration. Reproduisant des numéros du théâtre de variétés, ils contiennent tous les éléments de ce cinéma "exhibitionniste» dont parle Tom Gunning: la frontalité, les adresses à la caméra et, surtout, le salut au public des deux frères dans le dernier film de leur petit programme qui, significativement, porte le titre "Apothéose». Toutefois, le contexte historique des présentations fait en sorte que c'est encore le phénomène des images en mouvement qui fascine les spectateurs. Du moins, c'est cela que l'on peut conclure des diverses réactions dans la presse:

À la fin du programme on passe à la petite scène où se trouve le Bioskop. Le technicien ingénieux utilise ici des photogrammes instantanés amusants et les présente en les agrandissant. Ils ne sont pas fixes, mais vivants. Le diable saura comment il y arrive ${ }^{13}$.

Avec leur Bioskop, Messieurs Skladanowsky ont créé une invention extraordinaire: comparable au Elektrische Schnellseher, on transporte des artistes en action, par exemple des jongleurs, des acrobates, etc., sur la scène en grandeur nature. C'est étonnant ${ }^{14}$.

On pourrait citer d'autres exemples du même type. Ce qui frappe dans ce dernier exemple, c'est que le représenté, c'està-dire les numéros reproduits, n'est évoqué qu'en passant. De toute évidence, les auteurs de l'époque sont d'abord fascinés par les possibilités de la machine et ils expriment leur admiration face au spectacle jugé "étonnant». Or, s'ils se montrent étonnés, 
c'est davantage par le miracle technique qui se produit devant eux que par ce que montrent les images en mouvement.

Apparaissent alors les grandes lignes du dispositif spectaculaire constitué aux environs de 1895 par les images en mouvement: participant à plusieurs séries culturelles, les machines exhibent en premier lieu une technologie capable de capter et de restituer du mouvement; celle-ci vise à étonner et à émerveiller le spectateur à même la démonstration de ses possibilités, où se trouve privilégiée une forme filmique dont le but principal est de produire un maximum de visibilité. L'exemple des films tournés par les Skladanowsky démontre par ailleurs qu'une telle conception de la notion de dispositif mène en fait à une approche pragmatique historique: le dispositif informe le cadre institutionnel général au sein duquel fonctionnent les films. Autrement dit, une même forme filmique peut fonctionner différemment d'un dispositif à l'autre ${ }^{15}$.

\section{La cinématographie comme dispositif du spectaculaire}

Le paradigme de la captation/restitution — tout comme le dispositif qui lui correspond - est presque exclusivement lié à la période de la novelty. Pour que la cinématographie puisse s'établir en tant que forme de spectacle, pour que les spectateurs aient le désir de revenir voir des images en mouvement, il fallait que le représenté lui-même devienne une attraction. C'est ce que Gaudreault et Marion appellent le "paradigme de la monstration" et ce que, plus généralement, on a pris l'habitude d'appeler le cinéma des attractions. Or, l'exemple de la production des frères Skladanowsky que l'on vient d'évoquer indique déjà que la frontière entre le dispositif du premier paradigme, c'est-à-dire celui lié à la captation/restitution, et le deuxième, est particulièrement flottante. Dès que la dominante se déplace du spectacle de la reproduction du mouvement en tant que tel au spectacle représenté, aux attractions filmées, on passe à un dispositif autre.

Comme on vient de le voir, Tom Gunning a d'abord défini le cinéma des attractions comme un dispositif qui s'oppose, presque terme à terme, au dispositif du cinéma narratif classique. Et en effet, de nombreuses recherches récentes ont exploré cet aspect 
des attractions fortement présent dans la production cinématographique du tournant du siècle. Qu'il s'agisse des diverses formes des films à trucs, des féeries, des vues de plein air, des films comiques, on ne saurait négliger l'insistance sur le spectaculaire, sur les attractions visuelles de toutes sortes. Les catalogues de l'époque nous livrent par ailleurs de nombreux exemples de descriptions de films soulignant, justement, tel ou tel aspect particulièrement spectaculaire, des "magnifiques apothéoses en couleurs" aux accidents extraordinaires des films comiques en passant par des spectacles naturels reproduits dans des vues de plein air: couchers de soleil, chutes d'eau sublimes, paysages exotiques. L'usage récurrent de formules de présentation, équivalents rhétoriques du geste indicatif, "voici» implicites lors de l'énumération des éléments saillants du film, apparaissent alors comme des "consignes de lecture ${ }^{16}$ ".

Là encore, force est de constater que tous les genres qu'on vient d'évoquer participent de différentes séries culturelles: le théâtre magique, les féeries sur scène, le cirque, la prestidigitation, le panorama, le diorama, la lanterne magique (avec, au sein de cette série-là, divers genres comme, par exemple, les images de voyage), etc. ${ }^{17}$. Or, d'un certain point de vue, on peut dire que la dimension du spectacle visuel — «spectacle» étant alors à prendre au sens fort - fonctionne comme une sorte de dénominateur commun pour toutes ces séries culturelles. Il s'agit avant tout de "donner à voir", beaucoup plus que de raconter, de démontrer ou de faire savoir.

Les caractéristiques de ce dispositif du spectaculaire dans le domaine de la cinématographie sont alors les suivantes: la technologie n'est plus en elle-même l'attraction principale (comme c'est le cas de la toute première période présentant la cinématographie comme dispositif spectaculaire); elle sert avant tout à montrer $d u$ spectaculaire, participant de la sorte à d'autres séries culturelles tout en utilisant les possibilités techniques du média (la captation/restitution, qui devient alors en partie révélation, mais aussi d'autres possibilités comme les trucages, la mobilisation de l'appareil de prises de vues, la mise en scène, etc.). Ce dispositif fonctionne différemment de celui privilégiant l'appareil et la technologie. On cherche à étonner et à émerveiller le 
public par le spectacle visuel, la forme filmique ayant maintenant pour but de le mettre en valeur de manière optimale. On retrouve alors les caractéristiques du "cinéma des attractions" tel qu’il a été défini par Tom Gunning.

\section{Conclusion}

Le présent texte visait à redéfinir la notion de dispositif, élaborée il y a une petite trentaine d'années, en la révisant sur deux points fondamentaux: d'une part, il s'agissait de l'" historiciser" radicalement, en abandonnant l'idée "qu'un même dispositif serait à l'origine de l'invention du cinéma et déjà présent chez Platon", comme le disait Baudry et, d'autre part, de déplacer la notion du terrain de la métapsychologie du spectateur vers celui d'une pragmatique historique. Dans une telle perspective, il appert que la productivité théorique de la notion du dispositif repose surtout sur la manière dont elle cherche à articuler le fonctionnement de la technologie, le positionnement du spectateur et la forme filmique. Ceci permet, par ailleurs, de situer les différents paradigmes décrits par André Gaudreault dans un contexte institutionnel plus large. L'étude des formes se trouve ainsi encadrée par une analyse d'orientation pragmatique.

Dans la toute première période de l'histoire des photographies animées, on pourra alors distinguer deux fonctionnements de la cinématographie: d'une part la cinématographie comme dispositif spectaculaire, dans le cadre duquel c'est la reproduction du mouvement qui constitue le spectacle principal, et d'autre part la cinématographie comme dispositif du spectaculaire, où ce sont les attractions filmées qui doivent attirer le public ${ }^{18}$.

Pour terminer, il faudrait au moins formuler une nouvelle problématique induite par cette redéfinition de la notion que je propose.

Dans son introduction à un numéro thématique de la revue Film History consacré au cinéma des premiers temps, Stephen Bottomore (1998) utilise l'expression "Cambrian cinema" pour souligner l'extraordinaire richesse des formes filmiques et de leurs usages au début du XX $\mathrm{XX}^{\mathrm{e}}$ siècle. Or, la découverte de ces multiples phénomènes culturels nous pousse à raffiner davantage nos outils 
d'investigation. Devrons-nous recourir à la notion de "sousdispositif», pour décrire les diverses pratiques de présentation? On sait par exemple que dans des théâtres de variétés allemands, le film d'Edwin S. Porter, The Great Train Robbery (Edison, 1903), fut présenté comme une actualité ${ }^{19}$. Est-ce que cet événement fait en sorte que le film relève d'un autre dispositif ou en signale-t-il simplement une variante, n'affectant pas ce que j'appelle le dispositif du spectaculaire? Afin de tester leur efficacité heuristique, il faudra sans doute revoir les hypothèses avancées ici à la lumière de nombreuses études de cas. À ce stade de la recherche, je reste toutefois convaincu de leur valeur théorique, que ce soit pour l'analyse ou la modélisation de cette période riche et complexe que nous nommons toujours, faute de mieux, le cinéma des premiers temps.

\section{Universiteit Utrecht}

\section{NOTES}

1. Ce texte a été écrit dans le cadre de l'«Utrecht Media Research Project on the Historical Construction of Media and Media Forms ».

2. Théorie élaborée, on le sait, par Jean-Louis Baudry dans deux articles fondamentaux, repris par la suite dans Baudry 1978.

3. À noter que Christian Metz (1975, p. 132-133) insiste sur le fait que le régime spectatoriel qu'il décrit est, entre autres, culturellement spécifique aux pays occidentaux.

4. Cf. Baudry 1978 (p. 36) : "C'est dire aussi bien qu'un même dispositif serait à l'origine de l'invention du cinéma et déjà présent chez Platon. [...] On pourrait alors avancer que le "mythe" de la caverne est le texte d'un signifiant de désir qui hante l'invention du cinéma, l'histoire de l'invention du cinéma."

5. Tout en étant conscient du danger de procéder ici à des rapprochements superficiels et trop rapides, je voudrais signaler que le concept de dispositif, décrit de la sorte, a certains points en commun avec la notion de Ge-stell chez Heidegger, tout particulièrement là où le philosophe parle des machines non pas en termes d'objets, mais en termes d'un rapport complexe et réciproque entre la machine, la manière dont elle est utilisée et utilisable, et l'homme. Cf. Heidegger 1962 (notamment p. 14-20).

6. En Allemagne, Knut Hickethier a proposé de concevoir l'histoire du cinéma et de la télévision en tant qu'histoire de dispositifs ( $c f$. Hickethier 1995 et Hickethier 1997). Par contre, pour Siegfried Zielinski et son projet d'une histoire des "audio-visions", c'est justement le fait que le concept de dispositif lui permet de penser les grandes lignes d'une continuité à un niveau métapsychologique qui l'amène à travailler avec la théorie de Baudry (voir notamment Zielinski 1994).

7. Pour ma conception d'une pragmatique historique, voir Kessler 2000 et Kessler 2002. 
8. Sur Anschütz, voir notamment l'incontournable étude de Deac Rossell (2001), de laquelle je tiens toutes les données factuelles présentées par la suite.

9. Photographisches Wochenblatt, 1894, p. 424 (cité dans Rossell 2001, p. 120).

10. Cf. sur ce point aussi Gaudreault et Kessler 2002 (p. 24-25).

11. Cf. sur ce point Vogl-Bienek 1999 (notamment p. 96-99).

12. Voir l'annonce reproduite dans Zglinicki 1979 (p. 241 - notre traduction).

13. Staatsbürgerzeitung, $\mathrm{n}^{\circ}$ 519, 5 novembre 1895 (cité dans Zglinicki 1979, p. 242 - notre traduction).

14. Artist, $\mathrm{n}^{\circ}$ 561, 1895 (cité dans Zglinicki 1979, p. 242 - notre traduction).

15. Voir aussi l'article d'Uli Jung (2002) qui établit une distinction entre Lokalaufnahmen et Städtebilder, deux types de vues urbaines qui, d'un point de vue formel, peuvent apparaître identiques, mais qui different considérablement en ce qui concerne leur mode d'exploitation.

16. J'ai exploré diverses facettes de ce cinéma «attractionnel » dans différents articles. Voir Kessler 2000, 2000a, 2001 et 2003.

17. Il ne faut pas oublier, par ailleurs, les cas où des films apparaissent au sein d'un spectacle autre, comme par exemple certains films de Georges Méliès produits spécialement pour des féeries ou des revues.

18. L'analyse du discours journalistique à Montréal, effectuée par Martinez (2002), corrobore ces hypothèses de manière fort intéressante.

19. Cf. Garncarz 1998.

\section{RÉFÉRENCES BIBLIOGRAPHIQUES}

Aumont et Marie 2001 : Jacques Aumont et Michel Marie, Dictionnaire théorique et critique du cinéma, Paris, Nathan, 2001.

Baudry 1978: Jean-Louis Baudry, L'Effet-cinéma, Paris, Albatros, 1978.

Bottomore 1998: Stephen Bottomore, "The Cambrian Cinema", Film History, vol. 10, n 1,1998 , p. 3-7.

Elsaesser et Barker 1990 : Thomas Elsaesser et Adam Barker, "Introduction " à la section "The Continuity System. Griffith and Beyond", dans Thomas Elsaesser (dir.), Early Cinema. Space, Frame, Narrative, London, BFI, 1990, p. 293-317.

Garncarz 1998 : Joseph Garncarz, «Die Entstehung des Kinos aus dem Varieté: ein Plädoyer für ein erweitertes Konzept der Intermedialität», dans Jörg Helbig (dir.), Intermedialität: Theorie und Praxis eines interdisziplinären Forschungsgebiets, Berlin, Erich Schmidt Verlag, 1998, p. 244-256.

Gaudreault 1997 : André Gaudreault, "Les vues cinématographiques selon Georges Méliès, ou: comment Mitry et Sadoul avaient peut-être raison d'avoir tort (même si c'est toujours Deslandes qu'il faut lire et relire)... ", dans Jacques Malthête et Michel Marie (dir.), Georges Méliès, l'illusionniste fin de siècle?, Paris, Presses de la Sorbonne Nouvelle, 1997, p. 111-131.

Gaudreault et Kessler 2002: André Gaudreault et Frank Kessler, «L'acteur comme opérateur de continuité, ou: les aventures du corps mis en cadre, mis en scène et mis en chaîne", dans Laura Vichi (dir.), L'uomo visibile/The Visible Man, Udine, Forum, 2002, p. 23-32.

Gaudreault et Marion 2000 : André Gaudreault et Philippe Marion, "Un média naît toujours deux fois...", Sociétés et Représentations, n' 9, 2000, p. 21-36.

Gaudreault et Marion 2001: André Gaudreault et Philippe Marion, «Le cinéma naissant et ses dispositions narratives ", Cinéma \& Cie, nº 1, 2001, p. 34-41. 
Gunning 1990 : Tom Gunning, "The Cinema of Attractions. Early Film, Its Spectator and the Avant-Garde", dans Thomas Elsaesser (dir.), Early Cinema. Space, Frame, Narrative, London, BFI, 1990, p. 56-62.

Heidegger 1962: Martin Heidegger, Die Technik und die Kehre, Pfullingen, Verlag Günther Neske, 1962.

Hickethier 1995: Knut Hickethier, "Dispositiv Fernsehen», Montage/AV, vol. 4, $\mathrm{n}^{\circ} 1,1995$, p. 63-83.

Hickethier 1997 : Knut Hickethier, "Film und Fernsehen als Mediendispositive in der Geschichte", dans Knut Hickethier, Eggo Müller et Reiner Rother (dir.), Der Film in der Geschichte, Berlin, Sigma, 1997, p. 63-73.

Jung 2002: Uli Jung, "Local Views: A Blind Spot in the Historiography of Early German Cinema", Historical Journal of Film, Radio and Television, vol. 22, n 3, p. 253-273.

Kessler 2000 : Frank Kessler, «Regards en creux. Le cinéma des premiers temps et la construction des faits spectatoriels", Réseaux, n 99, 2000, p. 73-98.

Kessler 2000a: Frank Kessler, «In the Realm of the Fairies. Early Cinema between Attraction and Narration", Iconics. International Studies of the Modern Image, vol. 5, 2000, p. 7-26.

Kessler 2001 : Frank Kessler, "Cinématographie et arts de l'illusion», dans Leonardo Quaresima et Laura Vichi (dir.), La decima musa/The Tenth Muse, Udine, Forum, 2001, p. 535-542.

Kessler 2002: Frank Kessler, "Historische Pragmatik», Montage/AV, vol. 11, n 2, 2002, p. 104-112.

Kessler 2003 : Frank Kessler, "Mésaventures en série. La circulation des motifs dans le cinéma comique des premiers temps", dans Anna Antononini (dir.), Il film e i suoi multipli/Film and Its Multiples, Udine, Forum, 2003, p. 451-458.

Martinez 2002: Karine Martinez (en collaboration avec Jean-Pierre Sirois-Trahan), "La vue animée dans le discours journalistique (1896-1908)", dans François Albera, Marta Braun et André Gaudreault (dir.), Arrêt sur image, fragmentation du temps/Stop Motion, Fragmentation of Time, Lausanne, Payot Lausanne, 2002, p. 309-320.

Metz 1975: Christian Metz, "Le film de fiction et son spectateur», Communications, $\mathrm{n}^{\circ} 23,1975$, p. 108-135.

Rossell 1997: Deac Rossell, Ottomar Anschütz and his Electrical Wonder, London, The Projection Box, 1997.

Rossell 2001: Deac Rossel, Faszination der Bewegung. Ottomar Anschütz zwischen Photographie und Kino, Basel/Frankfurt/Main, Stroemfeld/Roter Stern, 2001.

Vogl-Bienek 1999: Ludwig Vogl-Bienek, "Skladanowsky und die Nebelbilder", KINtop. Jahrbuch zur Erforschung des frühen Films 8: Film und Projektionskunst, Basel/Frankfurt/Main, Stroemfeld/Roter Stern, 1999, p.83-100.

Zglinicki 1979: Friedrich von Zglinicki, DerWeg des Films, Hildesheim/New York, Olms Presse, 1979.

Zielinski 1994: Siegfried Zielinski, «Historic Modes of the Audiovisual Apparatus », Iris, $\mathrm{n}^{\circ} 17,1994$, p. 7-24. 